VI. Anordnung und Eintheilung der Meteoriten; com Freiherrn von Reichenbach.

\title{
IX.
}

Es ist noch nicht lange her, wenige Jahrzehnte erst, dafs wir kaum mehr als etwa ein Dutzend Meteoriten aufzuweisen wufsten. Beim Anfang unseres Jahrhunderts machten Ensisheim, Siena, Agram, Barbotan, Mauerkirchen, Yorkshire, Eichstädt, L'Aigle, Stannern, und einige andere den ganzen gesicherten Schatz aus, den wir als Geschenk des Himmels anerkannt und verstanden hatten. Das waren der zweifelfreien Thatsachen so wenige, und diese unter sich so verschieden, dafs daraus nur wenige verworrene Vorstellungen und all der unerquickliche Hader vorkommen konnte, mit dem unsere Erkenntnifs vom Wesen der Meteoriten begann.

Anders ist diefs gegenwärtig, wo wir in Wien allein 156 verschiedene Luftsteine zur Untersuchung vor uns liegen haben, und im Ganzen wohl an 200 in den verschiedenen Naturaliensammlungen der wissenschaftlichen Welt vorhanden seyn werden. Jetzt hat dic Erscheinung nicht blofs den Umfang gewonnen, indem sie sich in einer grofsen Anzahl von Vorkommnissen ausgesprochen hat, sondern sie bat auch den Inhalt erlangt, indem diese Vorkommnisse in mannichfaltigen Formen aufgetreten sind. Eine solche Anzabl von Objecten kann nicht mehr mit der Anschauung umfafst, noch weniger mit dem Gedächtnisse beherrscht werden; es ist daher nothwendig, dafs wir unsere Auffassung durch formale Hülfsmittel unterstätzen. Diefs geschieht durch die Anwendung der Eintheilung. Bis jetzt ist, meines Wissens, eine methodische Classification der Meteoriten und der in ihrem Gefolge auftretenden Erscheinungen nicht unternommen worden; man hat sich mit einer chronologischen Aufzählung der Fallzeiten oder mit einer trivialen Scheidung 
in Steine und Eisenmassen begnügt. Auch was Hr. Shepard versucht hat (Sillim. Journ. T. II Ser. II S. 377) ist von den europäischen Naturforschern nicht gebilligt worden (R a m m e l s berg's Handwört. Suppl. V, S. 38), und eine sogenannte Verwandtschaftstabelle der Meteoriien, die Partsch in seiner Schrift: "lie Meteoriten des k. k. Hofnaturalien Cabinets " aufgestellt hat, ermangelt einerseits eines Classificationsprincips, andertheils stützt sie sich auf unrichtige Begriffe. So theilt er die Meteorsteine ein in "anomale" und "normale". Was ist aber ein anomaler, was ein normaler Aërolith? Welches ist die Norm, die der Natur für einen Himmelsstein vorgeschrieben ist? das streift wieder an die bekaunte "Pathologie der Mineralien, "Mit welchem Grunde spricht man einer Anzahl Meteoriten normale Berechtigung ab und verweist sie so zu sagen in den Ausschufs des Anomalen? Gegen derlei Satzungen menschlicher Einbildung wehrt sich die Natur wie die Logik. Und unglücklicher Weise sind jene Meteoriten, denen Partsch den Rang der Normfähigkeit abspricht, wie Capland, Alais, Langres oder Chassigny $u$. a. gerade die interessantesten und wichtigsten, die wir haben. Ebenso ist seine Eintheilung in Steine und Eisenmassen nicht haltbar, da beide vielfach in einander verlaufen, ohne irgend eine Scheidegränze. Die Menge des angesammelten Materials aber, darin einerseits eine gewisse Einheitlichkeit seines allgemeinen Gepräges; anderseits eine Mannichfaltigkeit nach Form und Gehalt; die Aehnlichkeiten endlich, mit welchen die Individuen nach mancherlei Richtungen ihrer besonderen Ausbildung wieder unter einander sich nähern und gruppiren, Alles läuft allmählich so hoch an, dafs es uns durch eben so viele Momente zu geregelter Anordnung auffordert. Sie wird, nach naturhistorischen Grundsätzen entwickelt, uns gewisse Gesetze aufdecken, denen das Ganze wie die Einzelheiten der meteoritischen Erscheinungen unterstehen, und wird dazu beitragen, einige weitere Einblicke in ihr eigenthümliches Wesen zu thun.

Ein vollständiges und lückenlos zusammenhängendes 
System wird man indefs von diesem ersien Versuche nicht erwarten. Die Meteoriten zeigen sich unter einander so verschieden, manche davon stehen nach ihren Beschaffenheiten noch so isolirt, dafs 200 derselben noch nicht Stoff. genug gewähren, eine so weltumfassende Erscheinung nach ihrem ganzen Unfang darzustellen. Gleichwohl haben wir hinreichend Material zusammengebracht, um unter seinen Bestandtheilen Aehnlichkeiten hervortreten zu sehen, stark genug, um nach denselben Abtheilungen und Zusammenstellungen möglich zu machen. Wenn die Zahl der gewonnenen Meteoriten sich einmal auf einige tausend erhoben haben wird, und diefs kann vor Ablauf von zwei Jahrhunderten zu Stande kommen, dann werden wir Musterstücke von der ganzen Erscbeinung in allen ihren Gliedern besitzen und können sie vollständig darstellen. Für jetzt haben wir nur erst so viel, um zu übersehen, dafs sie in sich $\mathrm{Zu}$ sammenhang hat; dafs ihre Verschiedenheiten und Eigen. thümlichkeiten sich wiederholen, unter den verschiedensten Unständen auftreten, an den entlegensten Orten und zu den entferntesten Zeiten in gleicher Weise zum Vorchein kommen. Wir sind bereits im Stande, solche Wiederholungen mehrfältig zu sammeln und bëinahe idente Erscheinungen zusammenzubringen; wir vermögen Aehuliches in Gruppen zu vereinigen, und diese Gruppen zeigen wiederum der gemeinsamen Merkmale genug, um sie mit dem Bande ganzer Sippschaften umschlingen zu können.

Von diesen Gesichtspunkten ausgehend, will ich es nun versuchen, alle Meteoriten, die ich theils durch eigenen Besitz, theils durch autoptische Benutzung fremden Eigeuthums, namentlich der kaiserlichen Sammlung in Wien, näher kennen zu lernen Gelegenheit fand, zuerst in eine allgemeine Reihe zu bringen, welche fortlaufende Uebergänge von einem $\mathrm{zu}$ dem anderen zeigt, und in der das Material der ganzen Erscheinung obne Ausnahme untergebracht werden soll, danu aber nach einem Classificationsprincip mich umzusehen, das eine naturgemäfse Gliederung jener Reihe nach charakteristischen Merkmalen abzugeben im Stande wäre. 
Zum Behufe einer allgemeinen Reihe nun glaube ich am besten zu thun, wenn ich mich zunächst an das grofse Gesetz in der Natur, an die Polarität und den von ihr bedingten Dualismus aller Existenz halte. Alles in dem weiten Weltall huldigt dieser gewaltigen und obersten Norm alles Daseyns und so gebietet sie auch über den Bestand der Meteoriten. Und da wir an ihnen mit einem anorganischen Gegenstande zu thun haben, dessen Wesen in seinen elektrischen Verhältnissen, scinen Krystallisationskräften und seinen chemischen Verwandtschaften begründet ist, so wird es wohl nur

$$
\text { das elehtro-chemische Verhalten }
$$

seyn, das wir hier zum Eintheilungsgrunde für die Aufstellung einer klaren, geordneten und zusammenhängenden Reihe der gesammten Meteoriten wählen können.

Eine solche Reihe bildet sich, wenn wir die Aërolithen nach ihrem chemischen Bestande in elektronegative und elektropositive unterscheiden, mit dem einen Gliede von der stärksten Intensität des einen Poles des elektrischen Verhaltens beginnen, und durch alle zwischenliegende Glieder bis zu dem der gröfsten Intensität des anderen Poles fortschreiten.

Nun wissen wir, dafs die schweren Metalle sich alle elektropositiv gegen die Steine, gegen Sauerstoff, Schwefel, Kohlenstoff, Phosphor und andere verwandte Stoffe verhalten, die auf der negativen Seite stehen. Da dorthin namentlich das Eisen gehört, welches seiner Menge nach eine so vorwiegende Rolle bei den Aërolithen spielt, so gewährt uns dieses in vorliegender Beziehung einen brauchbaren Anhalt. Wir können ohne Bedenken sagen, dafs ein Meteorit um so mehr auf der elektropositiven Seite stehe, je mehr er im Verhältnifs zu anderen Bestandtheilen Eisen enthält. Aufser diesem ist es noch das Nickel, welches in einigermafsen erheblicher Menge regulinisch in den Meteoriten vorzukommen pflegt. Diese Metalle haben aber, das Eisen 7,7 das Nickel 9,0 specifisches Gewicht, während die sauerstoff - und schwefelhaltigen Steine der Meteoriten nur 
$2 \frac{1}{2}, 3$, bis gegen 4 wiegen. So finden wir dann, dafs bei der bekannten Zusammensetzung der meisten Meteoriten ihr elektrochemisches Verhalten um so negativer wird, je weniger sie specifisches Gewicht und je mehr sie blofs Steinbestand, d. i. Oxyde, dagegen um so positiver, je mehr sie specifisches Gewicht und freien regulinischen Metallbestand haben. So gelangen wir zu dem Vortheile, uns einfach an das specifische Gewicht der Meteoriten halten und ihr polares Verhalten geradezu voñ dem specifischen Gewichte ableiten zu können. Je geringer diefs ist, desto negativer wird sich ein Meteorit verhalten, und je gröfser, desto positiver wird er seyn.

Wohl dürfen wir nicht aufser Acht lassen, dafs die specifischen Gewichte, wie wir sie von den meisten Meteoriten von verschiedenen Beobachtern besitzen, nur innerbalb eines gewissen Spielraunes Genauigkeit und Richtigkeit haben können und deswegen nirgeuds als absolut zutreffend genommen werden dürfen. Kein Meteorit, nicht ein einziger, ist in allen seinen Theilen gleich; er ist ein breccienartiges Gemenge; ja er ist oft auf ganze kurze Abstände ganz ungemein verschieden. Das Eisen ist sehr ungleich darin vertheilt, häuft sich da und dort in einem Steinmeteoriten an, bildet einzelne Klümpchen von Hirsekorn bis Erbsengröfse, wie G. R os e im Meteorit von Wenden und ich in dem von Blansko gefunden haben; oder die Steine sind porös, bilden bisweilen viele kleine Höhlen wie Richmond, Jnvenas u. a.; oder sie sind ungleich dicht, bald zerreiblich und fast schwammig, gleich dichtem Bimssteine, wie Lontalax, bald dicht und steinig, wie Stannern, bald schliefsen sie eine Menge lockererer oder festerer Bröckchen ein, wie Mässing. Je nachdem nun Behufs der Abnahme des specifischen Gewichtes da oder dort ein Bruchstückchen abgezwickt wird, wird das Ergebnifs der Wägung ein verschiedenes seyn, und diefs kann oft bedeutend wechseln. Ebenso sind Eisenmeteoriten in der Regel ein bewunderswerthes Geflechte von krystallinischen Bildungen verschiedener metallischer Legirungen, von ungleich eingesprengtem Chromeisen, Schwefeleisen, 
Phosphoreisen, Graphit und olivinartigen Körpern. Hierin liegt dann der Grund, warum die Gewichtszahlen verschiedener Beobachter nirgends so wenig übereinstimmen, als bei Meteoriten. Diefs hat mich bewogen, mich bei Aufstellung der elektro-chemischen Reihe zwar in der Hauptsache an das specifische Gewicht zu halten, jedoch da und dort einige Decimalen der natürlichen Verwandtschaft der Steine unterzuordnen.

Da wo die specifischen Gewichte noch nicht bekannt: sind, habe ich die ungewogenen Luftsteine denen von bekanntem Gewichte angereiht, mit welchen sie die meiste Aehnlichkeit des chemischen Bestandes oder des äufseren Ansehens haben.

Noch habe ich da, wo mir die Uebergänge nicht hinreichend stetig $z u$ seyn scheinen, sondern wo ich eine Lücke oder einigen Sprung zu erkennen glaube, einen kleinen Abstand in der Aufstellung offen gelassen. Die Zukunft, die uns noch tausende von Meteoriten schenken wird, füllt ohne Zweifel diese Lücken nach und nach aus, und ehe ein Jahrhundert vergeht, werden sie gründlich und vollständig getilgt seyn.

Unter diesen Vorbehalten und Einschränkungen will ich es nun wagen, die mir bekannten Meteoriten sämmtlich in folgende Reihe zu bringen, welche von der Spitze des negativen Poles bis zu der des positiven fortschreitet.

Elektrochemische Reihe der Meteoriten.

Specifisches Gewicht.

1,70 Alais.

2,69 Capland.

3,11 Bishopville.

3,08 Jonzac.

3,07 Stannern.

3,17 Constantinopel.
Specifisches Gewicht.

3,11 Juvenas.

3,11 Ohaba.

3,07 Lontalax.

3,09 Nobleborough.

3,17 Bialystok.

3,21 Mässing. 
Specifisches

Gewicht.

3,39 Siena.

Kaba.

3,26 Renazzo.

3,24 Borkut.

3,33 Pultawa.

3,36 Benares.

Clarac.

Little Piney.

3,47 Richmond.

3,39 Parma.

3,39 Oahu.

3,37 Poliz.

3,40 Zaborzica.

3,42 Bachmut.

3,45 Mauerkirchen.

3,46 Forsyth.

3,47 Lucé.

3,47 Salès.

3,48 Apt.

3,49 Charkow.

3,49 Kuleschofka.

Czartorya.

Chandakapur.

3,50 Lissa.

Garz.

Oesel.

Aumières.

3,53 Glasgow.

Kakova.

3,54 Milena.

3,58 Hartfort.

3,58 Nashville.

Kikina.
Specifisches

Gewicht.

3,55 Vouillé.

3,55 Langres.

Macerata.

3,44 Mainz.

3,47 Chantonnay.

3,43 L'Aigle.

3,49 Krasnoi-Ugol.

Cereseto.

3,48 Ensisheim.

3,50 Madaras.

3,53 Bremervörde.

3,53 Weston.

3,54 Gütersloh.

3,55 Kursk.

Favars.

3,47 Slobodka.

Esnaude.

3,49 Berlanguillas.

3,54 Château-Renard.

3,55 Divina.

3,57 Utrecht.

3,60 Timochin.

3,60 Horzowitz.

3,60 Eichstädt.

3,61 Yorkshire.

3,61 Agen.

3,63 Doroninsk.

Killeter.

Okaninah.

3,64 Tipperary.

3,65 Limerick.

3,66 Liponas.

Poggendorff's Annal. Bd. CVII. 
Specifisches

Gewicht.

3,66 Lixna.

3,54 Simbirsk.

3,64 Erxleben.

3,70 Wenden.

3,66 La Baffe.

3,66 Nanjemoy.

3,66 Asco.

3,62 Barbotan.

3,63 Sigena.

3,63 Cabarras.

3,65 Tabor.

3,70 Blansko.

Heredia.

3,70 Wessely.

3,72 Grüneberg.

Tunga.

3,71 Seres.

3,71 Charsonville.

3,73 Macao

3,73 Toulouse.

3,76 Girgenti.

3,77 Ekatarinoslaw.

4,61 Hainholz.

$\left.\begin{array}{ll}\text { 6,52 } & \text { Bitburg } \\ 6,56 & \text { Sachsen }\end{array}\right) \underset{\text { बें }}{6,95}$

6,95 Pallas

7,05 Atacama

7,58 Brahin

$(5,00$ ?)
Specifisches

Gewicht.

$$
\mathbf{7 , 6 7 ?}\left\{\begin{array}{l}
\text { Tejupilco. } \\
\text { Xiquipilco. } \\
\text { Ocatitlan. } \\
\text { Istlahuacan } \\
\text { Manji. } \\
\text { Bata. }
\end{array}\right.
$$

6,50 Tarapaca.

6,82 Claiborne.

6,83 Salt-River.

7,07 Union County.

7,10 Ruff.

7,26 Cosby.

7,30 Tazewell.

7,30 St. Rosa.

7,34 Seneca.

7,38 Misteca.

Sevier.

Caryfort.

7,50 Cap.

7,55 Rasgata.

7,55 Zacatecas.

Kamtschatka.

Chester.

7,56 Tucuman.

Smithland.

7,64 Caille.

7,65 Bohumiliz.

Bruce.

7,66 Seeläsgen.

7,67 Guilford.

7,69 Putnam.

7,71 Hauptmanusdorf.

7,72 Senegal.

7,72 Burlington. 
Specifisches

Gewicht.

7,73 Lénarto.

7,74 Elbogen.

7,76 Petropawlowsk.

7,77 Schwetz.

7,81 Arva.

7,82 Agram.

7,82 Red-River.

7,82 Texas.
Specifisches

Gewicht.

Lockport.

Carthago.

7,88 Bemdego.

7,88 Durango.

Madoc.

Löwenflufs.

Charlotte.

7,90 Ashville.

Der polaren Reihe, in der wir nun hier sämmtliche mir zugänglich gewordene Meteoriten vor uns sehen, und ihrer mit aller Sorgfalt und vielfachen Prüfungen hergestellten Anordnung sind wenige Erläuterungen hinzuzufügen.

Zunächst wird man den Meteoriten von Simonod darin vermissen; ich habe mich von seiner Aechtheit nicht überzeugen können und halte ihn nicht für Gegenstand dieser Untersuchung. Er trägt keine Merkmale eines Aërolithen an sich, und sein Herkommen ist nach Pogg. Ann. Bd. 36, S. 562 und Bd. 37, S. 460 so schlecht beglaubigt, dafs ich diesen fremdartigen schwarzen Körper für einen Meteoriten nicht anerkennen kann. Hr. D uma s hat zwar vor $22 \mathrm{Jah}$. ren in der Pariser Academie seine Untersuchung übernommen, sie ist aber niemals erschienen (L'Inst. No.141, pag. 17).

Der Meteorit von Alais hat zwar Berzelius (Pogg. Ann. Bd. 33, S. 118) bei der trockenen Destillation aus einem ausgelaugten und wieder getrockneten Rückstande einiges wenige Wasser gegeben; doch beträgt der daraus berechnete Wasserstoff kaum über $\frac{1}{2}$ Proc. des Gewichtes vom ganzen Steine, kann also auf seinen Rang in der elektrochemischen Reihe nur geringen Einflufs nehmen und ihn in keinem Falle aus seiner Stellung verdrängen.

Das specifische Gewicht, das in Partsch "Meteoriten " S. 150 von Atacama, Pallas, Brahin, Sachsen und Bitburg im Mittel mit 6,94 angegeben ist, kann nicht vom Gesammtgewichte jedes dieser Aërolithen verstanden seyn und geht nur den Eisenbestandtheil davon an; diese, jeder in seiner 
Gesnumbtheit, müssen bei weitem leichter seyn, und können 5 nicht übersteigen. Aehnlich rerhält es sich mit den sechs Eisemmassen aus dem Tolucathale, die mit Einrechnung ihres Stein-, Graphit-, Schwefel-, Cbloreisengehaltes nicht über 6,50 Gesanmitgewicht sich erheben könuen.

La Baffe, Nanjemoy und Asco stehen wahrscheinlich ctwas zu lief in der Reihe. Ihrem äufseren Verhalten nach sollten sie bei Little. Piney erscheinen. Ob ihr specifisches Gewicht, das sie so tief in der Reihe hinabrückt, ganz genau sey, wird dadurch einigermafsen zweifelhaft.

Borkut wird mit einem specifischen Gewichte von 5,24 angegeben (Liebig's Jahrb. 1856, S. 912). Diefs ist unmöglich und wird ein Druckfehler statt 3,24 seyn.

Von dieser allgemeinen Anordnung aller Meteoriten zu einer Gesammtreihe haben wir uns zu einer Eintheilung derselben, zu einer Lösung in Theile nach naturwissenschaftlichen Bestimmungggründen zu wenden. Ein oberstes Princip für dieselbe aufzustellen, ist weniger leicht, als es viclleicht scheinen möchte. Wir haben es hier mit einem $\mathbf{G e}$ genstande zu thun, der nicht nur nicht einfach, sondern in höherem Grade zusanmengesetzt ist, als die meisten anderen Naturkörper. Ein Meteorit ist in den meisten Fällen eine überaus verwickelte Zusammensetzung, erst hervorgehend aus den Wirkungen der chemischen Affinität der ins Gemische eingeführten entfernteren Elemente; dann aus deren Producten, den componirenden Mineralspecies; endlich aus dem Gemenge der näheren Bestandtheile, auf welche alle zuletzt noch die Kraft und die Weise der mechanischen Aggregation Einflufs übt. Aus dieser vielfachen Synthese herans in rückläufiger Construction den Weg zu den bestimmenden Bedingungen zu finden, die dann als Eintheilungsgrund in Anwendung gebracht werden können, ist sicherlich weniger leicht, als bei jeder anderen naturwissenschaftlichen Disciplin descriptiver Art. 
Das Natürlichste wäre nun wohl, mich an die Mineralspecies zu halten, aus denen die Meteoriten zusammengesetzt sind, und dann mich damit an ein Mineralsystem anzuschliefsen, das die öffentliche Gutheifsung besitzt. Allein damit würde ich, soweit die Sachen bis heute vorgerückt sind, nicht weit kommen. Wir haben noch so wenige Zergliederungen der Meteoriten in ihre näheren Bestandtheile; das was uns der Fleifs Howard's, Berzelius', $H$, und $G$. Rose's, Bergemann's, Rammelsberg's geliefert hat, ist noch so wenig zablreich und zum Theil selbst noch, nach des Letzteren eigenem Ausspruche so unsicher, dafs an eine Verwendung im Sinne einer Classification für jetzt nicht gedacht werden kann. Andere, etwa physische Merkmale, kommen uns nicht zu Hülfe, wenigstens keine von durchgreifendem Charakter.

Es wird daher kaum etwas besseres übrig bleiben, als den Weg einzuschlagen, den die Botaniker betraten, als sie das natürliche System in ihre Doctrin einführten. Noch heute, wie damals, hält man ihnen entgegen, dafs sie hein oberstes Classificationsprincip für ihr System besitzen, und drängt man sie un Erklärung, so gestehen sie zu, dafs ihre Eintheilung in natürliche Familien auf nichts Gründlicheren, als auf einem allerdings geistreichen Tatonnement beruhe. Das heifst, man sucht und findet nach dunkeln Begriffen das Aehnliche zusammen, schnürt es in Bündel und nennt es eine natürliche Familie. Wir wollen hier das Recht, in welches die Pflanzenleute sich gesetzt haben, für unsere ebenfalls nur descriptive Aufgabe in Anspruch nehmen und für unsere Eintheilung der Meteoriten der Bequemlichkeit des Tatonnements uns theilhaftig machen.

Dem zufolge schlage ich vor, die Meteoriten in Sippen einzutheilen und diese in Gruppen zu zerlegen. Bei den heutigen Mineralogen ist es häufig üblich geworden, sich für ihre Eintheilungen der Ausdrüche Familie und Geschlecht zu bedienen. Allein diese Bezeichnungen gehören der organischen Welt an, wo die Zeugungsverwandten geschlechts- 
und familienweise zusammengehören; ein Stein wird wohl erzengt, aber nicht gezeugt, und kann deswegen weder dem Geschlechte seiner Eltern, noch der Familie seiner Anverwandten angehören. Ich glaube daher, für die Luftsteine keine Geschlechtsregister eröffnen zu sollen. Auch ist die Zusammensetzung eines Aërolithen zu wenig bestimmt und zu ungenau begränzt, um auf ihr auch nur die Bezeichnungen von Gattung und Art mit Berechtigung anwenden zn können. Ich bediene mich also der Worte Sippe und Gruppe, als einer Bezeichnung von der ungebundensten Art, mit deren dehnbarem Bande man alles umschlingen kann, was einigermafsen durch Aehnlichkeit zu freier Vereinigung geeignet erscheint.

So schlage ich dann für den Gesammteintheilungbegriff: Meteorit, Aërolith, Bätylie, Himmelsstein, Luftstein, als Classenbezeichuung vor den Ausdruck, mit dem ich beginne als

\section{Erste Sippe.}

In diese nehme ich die leichteren, von Gediegeneisen freien, mehrentheils weifslichen, weifsen oder hellgrauen mit glasglänzenden Rinden versehenen Meteoriten auf. Als Prototyp davon betrachte ich den Stein von Langres, auch Chassigny genannt. Er hat einen schwachen Stich ins gelblich Grünliche, ist aber sonst von ungewöhnlich einfacher Beschaffenheit, fast ohne Beigemenge, ein einfaches Mineral und verdiente als solches die gröfste Aufmerksankeit unserer Scheidekünstler, da man von seiner Einfachheit auf die Zusammenselzung anderer Meteoriten gründliche Schlüsse machen kann. Ihm zunächst reiht sich der herrliche Stein von Bishopville an, der in grofsen Partien mit Schneeweifs prangt und darauf durchsichtige farblose Rinde wie Glas zeigt. Die ganze Sippe ist nur klein und besteht aus folgenden wenigen Fallorten (Localitäten)

1. Gruppe:

Langres.

Bishopville.

Jonzac, Uehergangsglied. 
2. Gruppe:

Juvenas.

Stannern.

Constantinopel.

Letztere drei sind sich so anfserordentlich ähnlich nach dem äufseren Ansehen, dafs sie selbst das Auge des Kenners kaum zu unterscheiden vernag. Damit stimmt auch nach Hrn. Rammelsberg's sorgfältiger Analyse der chemische Bestand überein, der zwischen Juvenas und Stannern kaum noch einigen Unterschied zeigt; Constantinopel aber ist ihnen bis zur Verwechslung gleich.

\section{Die zweite Sippe}

besitzt weifsliche, bis ins blafsbläuliche ziehende Grundmasse, einiges jedoch weniges Gediegeneisen, häufiger fein eingesprengtes Schwefeleisen, ist meist etwas locker, ofters mehr oder minder zerreiblich, reich an fremdartigen Einschlüssen. Ihre Rinde ist, die Gruppe Lontalax ausgenommen, matt. Prototypisch sind die Steine von Mauerkirchen, Milena, Nashville oder Yorkshire, die in den Exemplaren, die ich davon besitze, kaum zu unterscheiden sind. Es gehören hieher, von dem feinern Korne zum gröbern fortschreitend: 


\section{8}

1. Gruppe.

Ohne Einschlufs von deutlichen dunkeln Kügelchen, höchstens hier und da ein einzelnes zerstreut:

Macerata.

Vouillé.

Nashville.

Bachmut.

Mauerkirchen.

Glasgow.

Kuleschofka.

Zaborzica.

Hartfort.

Czartorya.

Milena.

Yorkshire.

Forsyth.

Politz.

Aumières.

Chandacapur.

Kikina.

Oesel.

Charkow.

Ekatarinoslaw.

Kakova

Garz.

Apt.

Asco.

Slobodka.

Château-Renard.

Toulouse.

Girgenti.

Lissa.

Killeter.

Oahu.

Cereseto.

Favars.

bläuliche Unterart

Weifsliche
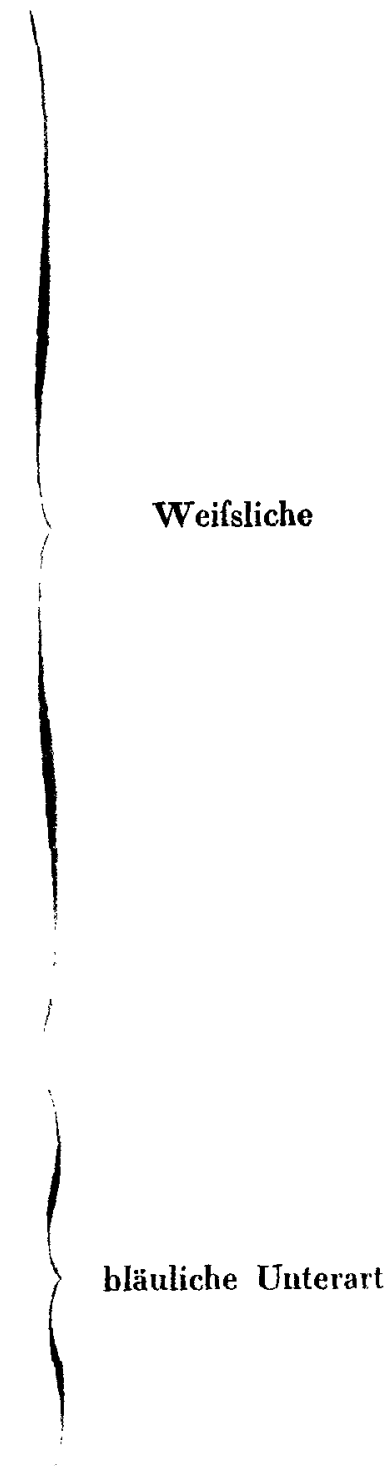
2. Gruppe.

Kügelchen einschliefsend und davon grobkörnig:

Salès.

Parma.

Lucé.

Nanjemoy.

Clarac.

Benares.

Utrecht.

Little Piney.

La Baffe.

Timochin.

Divina.

Horzowitz.

Eichstädt.

Richmond.

Pultawa.

Siena.

Lontalax.

Nobleborough.

Bialystok.

Mässing.
Uebergang

mit dunkelen

Kügelchen

Diese Sippe ist die bei weitem zahlreichste; die erste Gruppe besonders wiederholt sich so oft, dafs man sie als die gewöhnlichste Sorte von Meteorsteinen ansehen mufs. Die meisten davon sind sich untereinander so ähnlich, dafs selbst das Kennerauge sie auf dem Bruche oftmals nicht von einander zu unterscheiden in Stande ist. Dahin gehören die Steine von Nashville bis Asco, deren nicht weniger als vier und zwanzig an der Zahl. Am Ende nimmt die Gruppe einen Stich ins Bläuliche an, der wohl nur eine Spur von Grau ist. Das feinste und reinste Glied davon ist Macerata.

In der zweiten Gruppe werden die Steine auf dem Bruche rauh bis grobkörnig. In die weifse Grundmasse sind reichlich dunkelgraue Kügelchen cingelagert, die sich absondern und auflösen lassen und dann eine Kugelschale hinterlassen. 
Sie gehen von Staubfeinheit durch alle Abstufungen bis zu Erbsen - und Bohnengröfse. In der zweiten Art gesellen sich noch Kügelchen von andern Farben hinzu, hellgraue, hellbraune, hellgelbe u. s. w. Die vier letzten Aërolithen sind einander in den Handstücken, die ich sah, so aulserordentlich ähnlich, dafs ich aufser Stande bin, sie irgend von einander zu unterscheiden. Mein eigenes Exemplar von Lontalax verdanke ich der Gefälligkeit des Professors v. Bonsdorff zu Helsingfors, der es mir, unmittelbar abgebrochen von dem Hauptstücke bei der dortigen Universität, überschickte; es ist aufser allem Zweifel ächt. Und da deren wenig ins Publicum gekommen, der Stein daher sehr geschätzt ist, so ist an eine Fälschung bei den anderen nicht zu denken. "Desto auffallender und merkwürdiger mufs die bis zur Gleichheit gehende Aehnlichkeit von so hoch zusammengesetzten Luftsteinen seyn, wie die vier genannten es sind, man mufs bei ihrem Anblicke glauben, sie seyen alle von Einem Stücke abgeschlagen. "

Die dritte Sippe

sind die Meteoriten, die zunächst durch fein eingestreutes Eisenoxyduloxyd mit grauer Farbe ins Auge fallen. Sie sind fester, als die vorangegangenen, nicht zerreiblich, besitzen ungleich mebr Eisen, enthalten weniger Schwefelkies, und haben ein gröfseres specifisches Gewicht.

Sigena.

Macao.

Charsonville.

Esnaude.

Berlanguillas.

Liponas.

Gütersloh.

Weston.

Okaninal.

Fleckig

Tipperary.

Limerick.

L'Aigle.

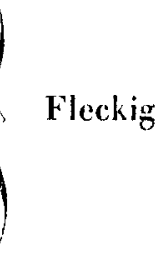


Seres.

Madaras.

Bremervörde.

Agen.

Doroninsk.

Lixna.

Cabarras.

Grüneberg.

Heredia.

Blansko.

Tabor.

Barbotan.

Wessely.

Krasnoi-Ugol.

Kursk.

Tunga.

Dunkelgrau

Ohaba.

Borkut.

Die drei erstern sind hellgrau; beide folgenden mittelgrau, feinkörnig; dann folgen die Gefleckten, deren Grundmasse bald bläulichweifs, bald grau in scharf abgegränzten gröfseren Flecken ist, so dafs sie das Aussehen haben, als ob Bruchstücke dieser und der vorangehenden Sippe zasammengekittet wären; sie bilden dadurch den Uebergang zwischen beiden; diefs ist besonders bei den drei ersten, von Liponas bis Weston der Fall. Von Okaninah bis L'Aigle sind die Flecken marmorartig wie unflossen. Seres bis Bremervörde schliefsen weifsliche Trümmer in dunklen Grunde ein. Agen und Doroninsk haben gleichfarbig graue Flecken. Darauf kommen elf ähnliche dunkelgraue Steine. Den Beschlufs machen zwei einfarbig tiefgraue Metcoriten.

\section{Die vierte Sippe}

unschliefst die grünen Luf(steine. Die chemische Ursache 
dieser Färbung ist noch nicht ermittelt und könnte von einer Nickel- oder Chromverbindung oder - weniger wahrscheinlich - von Eisenoxydul herrühren. Sie sind

Ensisheim

Simbirsk

$\left.\begin{array}{l}\text { Wenden } \\ \text { Erxleben }\end{array}\right\}$ Sehr ähnlich

Auch Charsonville und Oahu, die früher eingereiht sind, haben einen Stich ins Grüne; ebenso Hainholz. Ensisheim steht einigermafsen vereinzelt da; es findet nicht seines Gleichen unter den Meteoriten. Am nächsten kommt ihm noch Simbirsk, aber der Abstand bleibt inmer noch merklich. Wenden und Erxleben sind sich einander bis zur Gleichheit ähnlich und kaum zu unterscheiden. Simbirsk steht ihnen sehr nahe, entbehrt aber ihres Glanzes.

\section{Die fünfte Sippe}

soll die stark kohlehaltigen Meteoriten vereinigen. Sie sind alle schwarzbraun und schwarz, und zeigen damit, dafs es nicht blofs Kohlenstoff, sondern wirklich Kohle ist, die in ihnen eine so vorragende Rolle spielt. Die seltenen hiehergehörigen Fallorte sind:

\section{Alais. \\ Capland. \\ Kaba. \\ Renazzo.}

und bilden eine gut ausgesprochene Reihe von undeutlicher zu deutlicherer Ausbildung.

\section{Die sechste Sippe}

nimmt einige wenige Steine auf, welche in ungewöhnlicher Weise derbe, gröfsere braune Antheile enthalten, die kein Erzeugnifs der Verrostung seyn können, nämlich

Chantonnay.

Mainz.

Chantomnay ist von Berzelius aufs sorgfältigste untersucht 
worden; er läfst uns aber im Ungewissen über die Ursache der tiefbraunen Färbung, welche grofse über ihn verbreitete Flecke in so eigenthümlicher Weise enthalten '). In Mainz wiederholt sich dieselbe Erscheinung, die nicht von späterer terrestrischen Verwitterung herrühren kann, sondern in der ursprünglichen Beschaffenheit des Gemenges liegt. Dieser gleicht jenem in mehrfachem Betrachte und sieht äufserlich mehr einem armen Brauneisensteine, als einem Meteoriten ähnlich; im Wiener kais. Mineralien-Cabinete fand ich ihn durch dessen Vorstand von den Meteoriten ausgeschlossen, als unächt erklärt. Ein einfacher Anschliff zeigt was er ist: ein wohlcharakterisirter Meteorstein, der nicht verworfen werden kann.

\section{Die siebente Sippe}

erstreckt sich über Meteoriten, die durch Vermengung von steiniger Substanz und regulinischem Eisen die Zwischenglieder zwischen Steinmeteoriten und Eiscnmeteoriten bilden. Sie sind der Reihe nach von Stein zum Eisen fortschreitend, folgende:

1. Gruppe.

Atacama.

Pallas.

Sachsen.

Brahin.

Bitburg.

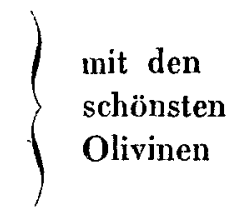

2. Gruppe.

Manji. Tejupilco.

Xiquipilco. die Tolucesen

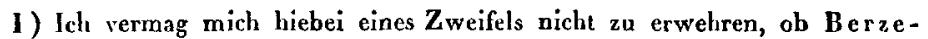
lius auch wirklich einen ächten Chantonnay bearbeitet habe. Er schildert ihn von Grundmasse theils schwarz, theils dunkelgrau mit schwarzen Streifen und ziemlich viel eingesprengtem Eisen. Das alles pafst nicht auf alle die Chantonnay, die ich gesehen habe. Die Grundmasse finde ich kastanienbraun, nirgends weder schwarz noch grau und ohne schwarze Streifen; eingesprengtes Eisen sehe ich verhältnifsmäfsig nar wenig, wie diefs auch das für einen so dichten Stein nur geringe specifische Gewicht von 3,47 bestätigt. Sollı Berzelius einen andern Meteoriten für einen Chantonnaỹ erhalten haben? Diefs wäre der Wichtigkeit seiner Analyse wegen ungemein zu bedauern und ein beruhigender $A$ ufschlufs hieriiher, wenn noch möglich, sehr zu wünschen. 
Hainholz trägt noch das äufsere Ansehen eines Steinmeteoriten, steht aber durch seinen hohen Eisengehalt und sein daraus hervorgehendes spec. Gewicht mit 4,61 (Wöhler) weit davon ab. Er unterscheidet sich von der ihm folgenden Pallaschen Gruppe nur durch gröfsere Feinheit seines Kornes. Diese begreift in sich alle die so sehr ähnelnden fünf Olivinträger, in zackiges Eisen eingefafst, das angeätzt eine eigenthümliche Art Widmannstettenscher Figuren mit sogenannten Fortificationszeichnungen besitzt.

Die sämmtlichen im Tolucathale gefundenen Eisenmeteoriten enthalten neben grofsen Graphiten und Schwefeleisen kleine, sofort bis bohnengrofse Körner von Olivin, und bilden den Uebergang zu den Eisenmassen.

\section{Die achte Sippe}

umschliefst die Eisenmassen mit Widmannstettenschen Figuren. Sie sind hier geordnet von den gröberen zu den feineren krystallinischen Bildungen fortschreitend, und sind sich so ähnlich, dafs sie nur Eine Gruppe ausmachen.

Mit Widmannstetten.

$\begin{array}{lll}\text { Seeläsgen. } & \text { Durango. } & \text { Caille. } \\ \text { Bemdego. } & \text { St. Rosa. } & \text { Lénarto. } \\ \text { Bohumiliz. } & \text { Buff. } & \text { Sevier. } \\ \text { Bruce. } & \text { Seneca. } & \text { Elbogen. } \\ \text { Union-County. } & \text { Carthago. } & \text { Ashville. } \\ \text { Cosby. } & \text { Schwetz. } & \text { Agram. } \\ \text { Madoc. } & \text { Texas. } & \text { Löwenflufs. } \\ \text { Misteca. } & \text { Lockport. } & \text { Tazewell. } \\ \text { Burlington. } & \text { Red-River. } & \text { Charlotte. } \\ \text { Guilford. } & \text { Petropawlowsk. } & \text { Putnam. }\end{array}$

Die krystallnischen Streifen von Putnam und Charlotte haben nahezu den achten Theil der Breite derer von Seeläsgen, Bemdego und Bohumiliz; die von Putnam erreichen 
kaum einen halben Millimeter Breite; während die von Bohumiliz vier, fünf, ja manche bis auf sechs Millimeter Breite sich verdicken; Putnam schaut sich so fein an, genau wie die Markstrahlen von Birnbaumholz auf der Spiegelseite; in Seeläsgen aber schwellen sie mitunter auf mebr als einen Centimeter an. Trotz ihrer schönen Krystallfiguren; ihrer Kiese und Graphite sind sie doch die gemeinen unter den Eisenmeteoriten, die immer nur dieselben Beschaffenheiten an sich tragen. Die nämlichen Figuren zeigt ein gröfseres Eisenkorn im Blansko-Steinmeteoriten.

\section{Die neunte Sippe}

vereinigt alle Eisenmeteoriten ohne Widmannstettensche Figuren und damit die mannichfaltigsten und interessantesten Bildungen, über welche wir die Aufschlüsse von der Zukunft hoffen müssen.

1. Gruppe. Uebergangsglieder.

Caryfort (de Kalb County).

Zacatecas.

2. Gruppe. Weifses Eisen in feinen Nadeln und Punkten.

Cap.

Rasgata.

Salt-River.

Kamtschatka.

3. Gruppe. Weifses Eisen in regellosen Klumpen. Chester.

Arva.

Caille (im kais. Cabinet)

4. Gruppe. Eisenmassen mit untergeordneten geradlinigen Schnitten.

Tucuman (Otumba.)

Senegal.

Mit regellosen Linien, Schnitten, Kiesflecken, Punkten glänzenden und trüben Stellen ohne erkennbare Ordnung: Claiborne. 
5. Gruppe. Ganz zeichnungslose auf der polirten angeäzten Oberfläche:

Tarapaca (Darlington.)

Green County.

(?) Hauptmansdorf (Braunau.)

Smithland.

Die erste Gruppe, in welcher Caryfort und Zacatecas sich befinden, ist nicht ohne gewisse Lineamente, welche den Widmannstettenschen Figuren einigermafsen entsprechen, aber in veränderter unausgebildeter, und durch die Verflechtung mit vielen Schwefelkiesen gestörter Weise.

Die zweite Gruppe mit Cap, Rasgata, Salt-River und Kamtschatka sieht nach dem Anätzen einem Meteoriten gar nicht ähnlich und die Eisen davon sind bisweilen gar nicht für Meteoriten anerkannt worden. Wenn man sie aber genau untersucht so gewahrt man äufserst feine weifsliche Nadeln und Fleckchen bald liegend, bald im Querschnitte als glänzende Punkte, welche von der Säure nicht angegriffen werden. Ihre chemische Constitution ist noch nicht erforscht.

Die dritte Gruppe mit Chester und Arva zeigt diefs weifsliche Eisen unordentlich durch die Masse zerstreut, nicht in regelmäfsigen Gestalten, sondern formlos klumpig eingelagert. Ein ähnlicher Fall tritt mit Cajlle ein; einige Exemplare sah ich ganz regelmäfsig mit Widmannstettenschen Figuren überzogen, eins aber im kaiserlichen Cabinet zeigt auf der Aetzfläche klumpiges Weifseisen 5 bis 6 Millimeter dick.

Die vierte Gruppe mit Tucuman und Senegal entwickelt zwar unter der Säure ein moirirtes Geflimmer, das man auch sonst zwischen den Widmannstettenschen Figuren öfters trifft, allein sonst keine regelmäfsige Bildung, wohl aber räthselhafte Einschnitte mitten im Eisen nach allen Richtungen.

Claiborne steht allein da. Die Beziehungen, welche unter seinen regelosen Linien, Schnitten, Kiesflecken u. s. w. stattfinden, sind noch ganz unerforscht. 
Die fünfte Gruppe mit Tarapaca, Green County, Haupt mannsdorf und Smithland fällt nach dem Aetzen durch völlige Formlosigkeit auf; doch hat man an Hanptmannsdorf bei vorsichtiger Behandlung feine dicht nebeneinanderliegende, gerade Linien entdeckt, die parallel durch die ganze Schnittläche laufen, änhlich den Nerven an einem monocotylen Blatte. Tarapaca enthält nach Darlington Kügelchen von regulinischem Blei.

In die interessante Mannichfaltigkeit und Verschiedenheit der Bildungen dieser Sippe erfordert es ein näheres Eingehen, um die Lehren daraus zu ziehen, die sie uns zur Beurtheilung ihrer Entwicklung geben; ich hoffe später darauf zurückzukommen.

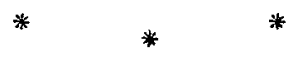

Schliefslich will ich die Gesammtheit der hier behandel. ten-Meteoriten, wie sie in meiner Sammlung vorhanden sind, nebst einer kleinen Anzahl aus dem kaiserlichen Hofmineralien Cabinet zu Wien, soweit sie mir zugänglich waren, nach der Ordnung der hier entwickelten Sippen und Gruppen gedrängt zu Einer Tafel zusammenstellen.

\section{Eintheilungstafel}

der Meteoriten der Reichenbach'schen Sammlung, naturhistorisch nach äufseren Merkmalen geordnet.

I. Sippe. Steine frei von regulinischen Metallen:

1. Gruppe. Langres.

Bishopville.

Jonzac. Uebergangsglied.

2. Gruppe. Juvenas.

Stanneru.

Constautinopel.

Poggendortf's Annai. Bd. CVII. 
II. Sippe; mit weifslicher Grundmasse.

1. Gruppe. Macerata.

Vouillé.

Nashville.

Bachmut.

Mauerkirchen.

Glasgow.

Kuleschofka.

Zaborzica.

Hartfort.

Czartorya.

Milena.

Yorkshire.

Forsyth.

Poliz.

Aumières.

Chandacapur.

Kikina.

Oesel.

Charkow.

Ekatarinoslaw.

Kakova.

Garz.

$\Lambda$ pt.

Asco.

Weifsliche mit

leichten Ein-

schlüssen.

Slobodka.

Château Renard.

Toulouse.

Girgenti.

Lissa.

Killeter.

Oahu.

Cereseto.

Bläulich weifsliche

Favars. 
2. Gruppe. Salès.

Parma.

Lucé.

Nanjemoy.

Clarac.

Benares.

Utrecht.

Little - Piney.

La-Baffe.

Timochin.

Divina.

Horzowiz.

Eichstädt.

Richmond.

Pultawa.

Siena.

Lontalax.

Nobleborough.

Bialystok.

Mässing.

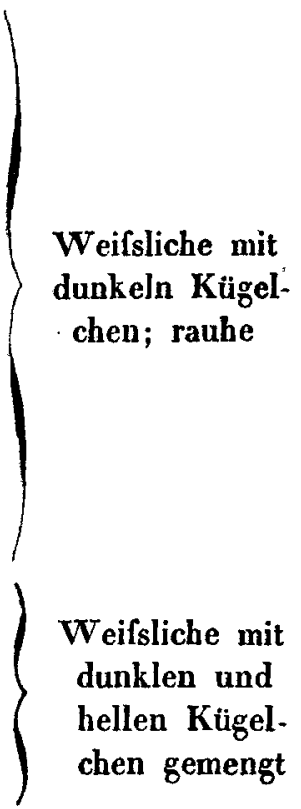

III. Sippe; Graue

Sigena.

Macao.

Charsonville.

Esnaude.

Berlanguillas.

Liponas

Gütersloh.

Weston.

Okaninah.

Tipperary.

Limerick.

L'Aigle.

Grau und weils gefleckte 
Seres.

Madaras.

Bremervörde.

Agen.

Doronisk.

Lixna.

Cabarras.

Grüneberg.

Heredia.

Blansko.

Tabor.

Barbotan.

Wessely.

Krasnoi - Ugol.

Kursk.

Tunga.

Ohaba.

Borkut.

IV. Sippe. Grünliche:

Ensisheim.

Simbirsk.

Wenden.

Erxleben.
Weirsliche Einschlüsse

Dunkelgrau

Sehr ảhnlich

v. Sippe. Die Kohligen:
Alais.
Capland.
Kaba.
Renazzo.

VI. Sippe. Die rostbraunen:

Chantonnay.

Mainz. 
VII. Sippe; die Mittelglieder zwischen Stein und Metall: Hainholz. Uebergangsglied

1. Gruppe. Atacama. Pallas.

Sachsen.

Brahin

Bitburg.

2. Gruppe. Istlahuacan.

Ocatitlan.

Bata.

Xiquípilco.

Tejupilco.

Manji.

Mit reinem

Olivine

Mit Steinein-

schlüssen

VIII. Sippe; die krystallinischen Metalle mit Leisten von Nickeleisen.

Seeläsgen.

Bemdego.

Bohumiliz.

Bruce.

Union - County.

Cosby.

Madoc

Misteca.

Burlington.

Guilford.

Durango.

St. Rosa.

Ruff.

Seneca.

Carthago.

Schwetz.

Texas.

Lock port.

Red-River. 
Petropawlowsk.

Caille.

Lénarto.

Sevier.

Elbogen.

Ashville.

Agram

Löwenflufs.

Tazewell.

Charlotte.

Putnam.

IX. Sippe; die Metalle ohne Widmannstetten'sche Figuren.

$\begin{array}{ll}\text { J. Gruppe. } & \left.\begin{array}{l}\text { Caryfort. } \\ \text { Zakatecas. }\end{array}\right\} \begin{array}{c}\text { Uebergangs- } \\ \text { glieder }\end{array}\end{array}$

2. Gruppe. Cap.

Rasgata.

Salt-River.

Kamtschatka.

Mit Nadeln und

Punkten

3. Gruppe. Chester.

Arva.

$\left\{\begin{array}{c}\text { Mit Klumpen } \\ \text { von Weilseisen }\end{array}\right.$

4. Gruppe. Tucuman.

Senegal.

Mit Schnitten

Claiborne.

unbestimmt

5. Gruppe. Tarapaca.

Green County.

Hauptmannsdorf.

Smithland.

innerlich

anscheinend

gestaltlos. 